\title{
Investigation of the Effects of Emphysema and Influenza on Alveolar Sacs Closure through CFD Simulation
}

\author{
Parya Aghasafari, Israr B. M. Ibrahim, Ramana M. Pidaparti* \\ College of Engineering, University of Georgia, Athens, GA, USA

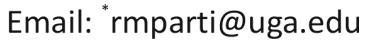

Received 17 December 2015; accepted 16 May 2016; published 20 May 2016

Copyright (C) 2016 by authors and Scientific Research Publishing Inc.

This work is licensed under the Creative Commons Attribution International License (CC BY). http://creativecommons.org/licenses/by/4.0/

\section{(c) (i) Open Access}

\begin{abstract}
Emphysema and influenza directly affect alveolar sacs and cause problems in lung performance during the breathing cycle. In this study, the effects of Emphysema and Influenza on alveolar sac's air flow characteristics are investigated through Computational Fluid Dynamics (CFD) simulation. Both normal and Emphysemic alveolar sac models with varying collapsed volumes resulting from influenza virus replication were developed. Maximum, area average pressure, and wall shear stress (WSS) in collapsed and open alveolar sacs models were compared. It was found that a collapse at half of the volume at the bottom of the alveolar sacs' models would cause a decrease in average and maximum pressure values and yield higher WSS values for fluid flow during the breathing cycle. On the other hand, a quarter volume collapse at the bottom and side of the model resulted in higher values for average and maximum pressure and WSS. Additionally, results also showed that a combination of alveolar sacs closure and Emphysema would generally lead to an increase in fluid pressure and average WSS during breathing. Maximum WSS was observed during exhalation and maximum WSS decrease occurred during inhalation. Findings are in good agreement with previous studies and suggest that emphysema and influenza virus affect fluid flow and may contribute to alveolar sac closure. However, more realistic simulations should include the fluid-solid interaction studies.
\end{abstract}

\section{Keywords}

Emphysema, Influenza, Wall Shear Stress (WSS)

\footnotetext{
*Corresponding author.
}

How to cite this paper: Aghasafari, P., Ibrahim, I.B.M. and Pidaparti, R.M. (2016) Investigation of the Effects of Emphysema and Influenza on Alveolar Sacs Closure through CFD Simulation. J. Biomedical Science and Engineering, 9, 287-297. 


\section{Introduction}

During mechanical ventilation, a sectional collapse of lung may occur when the lung air sacs become deflated and this condition is called Atelectasis. Some diseases including asthma, chronic obstructive pulmonary disease (COPD), and cystic fibrosis are associated with atelectasis. Emphysema is one of the main types of COPD that causes a gradual loss of air sacs wall surface area within the lung. This surface area loss would affect the absorption of oxygen. As the destruction of air sacs' structure progresses, there would be increasing difficulty in expelling the air during exhalation. Emphysema is considered to be the fourth leading cause of death in the United States based on statistics from the American Lung Association. Simulation of fluid flow under the effect of Emphysema would provide critical information for the respiratory specialists to prevent undesired injuries during mechanical ventilation (MV).

Computational fluid dynamics has been widely used over recent years to study different aspects of fluid flow related to the acinar region. Some studies employed CFD modeling and focused on flow patterns and effective parameters for assessing the influence of flow patterns on particle depositions and heat and mass transfer characteristics in alveolar cavities. Karl et al. [1] studied the effect of cavity geometry on flow field in the acinar region. They concluded that the ratio of duct volume to cavity volume would be an effective parameter of flow patterns in alveolar sacs. Sznitman et al. [2] considered rhythmic breathing motion of the pulmonary acinus and simulated induced air flow through the model of the alveolated ducts at each generation of the pulmonary acinar tree. They demonstrated the importance of three dimensional (3D) simulations in investigating existing complex flow patterns in alveolar sacs. In their study, it was determined that recirculation was highly dependent on geometrical changes in the model. Henry et al. [3] studied fluid particles' behavior in a realistic model of alveolated duct and considered rhythmically expanding walls for their simulation. They observed that wall expansion causes chaotic flow which would lead to kinematic irreversible flow in the acinar region. They determined this phenomenonas the origin of aerosol transport. Tsuda et al. [4] investigated airflow in the pulmonary acinus under the effect of rhythmic expansion of alveolar walls. They concluded that slight changes in alveolar wall motion would cause irreversibility and stretch-and-fold convective mixing in the acinar region that gave rise to deep penetration of ultrafine aerosol particles into the lung. Darquenne et al. [5] conducted CFD simulations on the acinar region of the human lung with expanding boundaries. They showed that geometric characteristics had a great impact on aerosol transport and deposition.

As discussed in the preceding paragraphs, geometric changes significantly affect fluid flow in alveolar sacs. Accordingly, some studies focused on lung diseases which are associated with alveolar collapse and atelectasis to explore their effects on fluid flow in the alveolar region. Otis et al. [6] studied alveolar pressure while mimicking collapsing that would happen during ventilation. They showed that there is a sudden pressure drop in the alveolar region due to a collapse in the respiratory system. Oakes et al. [7] studied fluid flow in 3D expanding, healthy, and Emphysemic alveolar sac models by using particle image velocimetry under realistic breathing conditions. Their study clarified that there is not irreversible flow in alveolar sacs in both healthy and Emphysemic models and that there is more particle sedimentation in the healthy alveolar sac model as compared to the Emphysemic model. Fitz-Clarke et al. [8] developed a computational model of the human respiratory system to study compression and re-expansion of airways and alveolar sacs during breathing. They concluded that a progressive alveolar collapse would increase hysteresis of pressure-volume loop and destroy the surface area for gas exchange. Schirrmann et al. [9] simulated human alveolar recruitment in normal and injured lungs and analyzed the interaction and stability in a system composed of two alveoli based on alveoli pressure-volume curves. They determined that injured alveoli show higher surface tension which would lead to a collapse in normal breathing pressure. Berg et al. [10] explored fluid motion and particle transport in healthy and Emphysematous alveolar sac models. They did not find recirculating flow in both models but they determined that the Emphysematous flow rate is three times larger than the flow rate in the healthy model. They pointed out that wall destruction under the influence of Emphysema would significantly affect fluid flow in alveolar sacs.

Apart from Emphysema, some other diseases have also been reported to affect alveolar sac's performance. For example, airways can be blocked by a foreign object or tumors that prevent the alveolar sacs from filling with air and collapse of lung tissue can occur in the affected area. Influenza viruses are among those foreign objects that cause alveolar sac closure and they are among the most common factors that lead to human respiratory infections and cause high morbidity and mortality rate [11]. Salmon et al. [12] investigated the influence of airway closure and alveolar collapse on transpulmonary pressure-volume curves under large-volume deflation and 
inflation. Tsuchida et al. [13] investigated contribution of atelectasis to ventilator-induced lung injury and they concluded that atelectasis leads to alveolar injury in the nonatelectatic lung.

Several studies focused on the effect of Emphysema and alveolar collapse on fluid flow through alveolar sacs separately but none of these studies focused on the effect of alveolar closure on Emphysemic alveolar sacs. In this study, a simplified bronchiole alveolar sac model was designed in Solid Works based on dimensions from real alveolar sacs models [14]. As Emphysema involves damage to the walls of the air sacs of the lung, it is assumed that the normal alveolar models which have a raspberry shape will change into a spherical form under the influence of Emphysema. CFD simulations are conducted on both models and results are compared to investigate the influence of Emphysema on fluid flow in human alveolar sacs. Next, the effect of Influenza virus on fluid flow was simulated. For this purpose, different volumes of alveolar sacs are occluded to mimic virus particle replication and fluid flow is post processed for different case studies and finally, the influence of collapse in Emphysemic alveolar sacs model is illustrated. This study can provide important information about the effect of two main medical disorders (Emphysema and Influenza virus replication) on fluid flow in alveolar sacs during the breathing cycle.

\section{Materials and Methods}

3D alveolar Sac Models were imported from Solid Works to ANSYS Workbench Fluent solver for generating the appropriate mesh and conducting the CFD simulation. Navier-stokes equations for laminar flow and second order implicit transient solver were used for the simulation. Inlet velocity boundary conditions for MV are loaded as user-defined functions (UDF) which are defined based on hospital data. Previous studies on flow in the lower airways showed that wall motion would not considerably affect fluid flow. Therefore, no-slip boundary conditions are applied at the entire model wall for simplicity [15]. Post processed pressure and wall shear stress (WSS) contours and velocity vectors at different locations through the models are extracted and results for different geometries are compared.

\subsection{Geometry}

Different geometric models have been considered for the alveolar studies, Fung et al. [16] assumed all alveoli are initially equal and space filling, and would uniformly ventilate and follow elasticity laws. Kitaoka et al. [17] used a four-dimensional alveolar model, corresponding to elastin fibers at alveolar mouths and junctions of alveolar septa to simulate alveolar deformation. Hansen et al. [18] studied different airway branching patterns and air spaces of a single human terminal bronchiole. Kumar et al. [19] considered honeycomb shapes for acinar geometries to investigate particle transport in these regions. Other studies also considered a polyhedral shape for alveoli models [20] [21]. Several 3D geometrical models of the alveolus airways have been previously completed. Some considered alveoli surrounded by cylinders [22] [23] or portions of annular rings [24]-[26], some studied 14-sided polyhedron models [27]-[29] and cylinder surrounded by spherical caps [30] [31]. In this study, an alveolar sac is designed in a raspberry shape based on dimensions from real alveolar sacs models. Since Emphysema causes damage to the air sac walls of the lung and leads to a slight volume increase in the alveolar model [7], the spherical model is designed to simulate the effect of emphysema on alveolar sac's fluid flow Figure 1.

\subsection{Governing Equations}

In this study, airflow in the acinar airways is assumed to be laminar and incompressible. Therefore the 3D incompressible laminar Navier-Stokes and continuity equations in a 3D mesh with a control volume approximation [32] are solved numerically to give the velocity field within the acinar region:

$$
\begin{aligned}
\nabla \cdot u & =0 \\
\rho\left(\frac{\partial u}{\partial t}+u \cdot \nabla u\right) & =-\nabla p+\mu \nabla^{2} u
\end{aligned}
$$

where $u$ is the velocity field, $p$ is the pressure, and $\mu$ is the dynamic viscosity. The continuity equation represents the conservation of mass, Equation (1), and the Navier-Stokes equations represent the conservation of momentum, Equation (2), that would be solved numerically on a moving grid using a commercial finite-volume based 


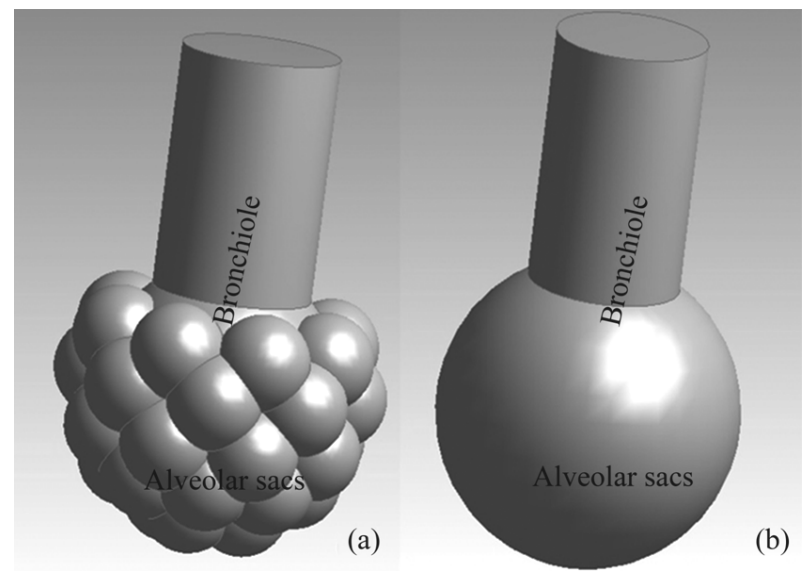

Figure 1. (a) Realistic alveolar sac model (b) alveolar sac under influence of Emphysema

program with fully implicit time marching techniques under isothermal conditions in ANSYS fluent solver [33].

\subsection{Boundary Conditions}

Boundary conditions define the inputs of the CFD simulation model. Some conditions, like velocity and volumetric flow rate, define how a fluid enters or leaves the model. When solving the Navier-Stokes equation and continuity equation, appropriate initial conditions and boundary conditions need to be applied. In this study, the no-slip boundary condition is applied at the solid wall and inlet velocity boundary condition is defined as $C++$ subroutine User Defined-Function (UDF) that is written based on hospital data. These conditions would be applied at the inlet area geometry for the whole model and the generation number would specify fluid velocity through the model. For this purpose, inlet velocity profiles are subsequently defined in the form of constants and exponentially decreasing flow rate profiles for inhalation and exhalation during mechanical ventilation are presented in Table 1. As presented in Table 1, velocity profiles are expressed as a function of the cross section (S), generation number $(g)$ (which is equal to 23 for alveolar sacs based on generation counting system from trachea to alveolar sacs as per [29]) and inhalation time $\left(t_{i n}\right)$ and exhalation time $\left(t_{e x}\right)$.

The flow rate $(Q)$, is defined as the proportion of tidal volume to inhalation time that is the lung volume that represents the normal volume of air displaced between inhalation and exhalation when extra effort is not applied. A tidal volume of $420 \mathrm{~mL}$ is considered in this study.

\section{Collapsed Alveolar Sacs}

Influenza virus replication causes bronchioles air flow closure. Once the sacs are internalized by respiratory epithelial cells, they replicate and lead to cell death and alveolar sac closure [34]. In this study, several alveolar sac collapse cases were studied to see how aggregation of influenza viruses diminishes alveolar sacs' volume during ventilation and would affect air flow under mechanical ventilation. For this purpose, three different cases were investigated; collapse at a quarter of the volume and half of the volume at the bottom of the alveolar sac and collapse at a quarter of volume at the side of the model, as presented in Figure 2. Next, CFD simulations were conducted on all cases with the same boundary conditions. Comparison of results between collapsed cases and open alveolar sacs and different collapse cases for normal alveolar sacs and alveolar sacs under Emphysema were completed to show how Emphysema and Influenza viruses would affect alveolar sac closure and impact fluid flow during ventilation.

\section{Results and Discussion}

Airflow characteristics obtained from CFD simulation for two alveolar sacs models are discussed next? Wall shear stress (WSS) contours and velocity vectors were extracted and pressure and WSS curves versus time were analyzed to evaluate the influence of Emphysema on fluid flow through an alveolar sac model. Additionally, the 
Table 1. Inlet velocity boundary condition.
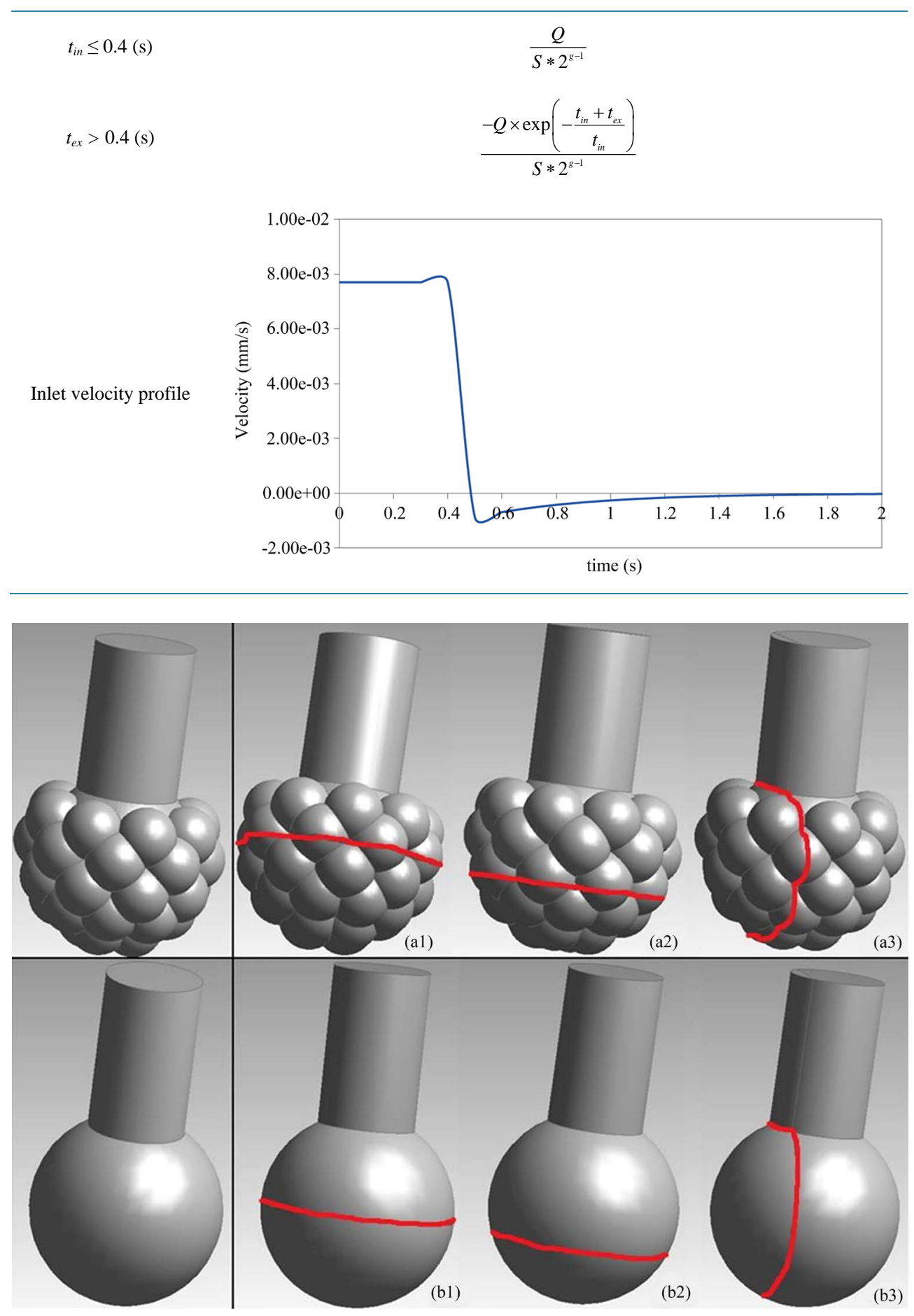

Figure 2. Collapsed case studies for Normal alveolar model (a1)-(a3) and Emphysemic alveolar sac models (b1)-(b3).

effect of alveolar volume closure on fluid flow during mechanical ventilation was investigated. For this purpose, fluid flow through collapsed cases was studied and compared with fluid flow in non-collapsed models for both normal and Emphysemic alveolar sac models to determine the effect of geometric changes and obstruction on fluid flow through alveolar sacs. 


\subsection{Velocity Vectors and WSS Distribution}

Velocity vectors for collapsed and open alveolar sac models are presented in Figure 3 at two different times (0.7 and 1.2 secs). As can be seen from the results shown in the figure, fluid does not move at the same velocity at every point in the alveolar sac. Based on no-slip condition applied on the wall, fluid velocity has zero magnitude on the surface and it will decrease with distance towards the inside of the model and inlet. The direction of the velocity is positive (into the models) during inhalation and negative (in inverse direction) during exhalation, which supports assumptions about inhalation and exhalation during ventilation. Also, as can be observed from Figure 3, Emphysema would lead to higher velocity values compared to normal alveolar sacs model.

The fluid velocities have a parabolic profile for the laminar flow regime. This flow pattern is related to existing friction between the fluid and the alveolar wall and fluid itself, which is relevant to the fluid viscosity. A tangential force would be generated through fluid flow by discussed friction and is known as the "wall shear stress". Wall shear stress's magnitude depends on how fast the fluid velocity rises when flowing from the alveolar wall toward the center of the alveolar sac. Wall shear stress for Newtonian fluids is defined according to Newton's law by the equation:

$$
\tau=\mu \cdot \frac{\mathrm{d} u}{\mathrm{~d} x}
$$

where $\mu, u, x$ and $\mathrm{d} u / \mathrm{d} x$ are the kinetic viscosity, fluid velocity, distance from the surface and the velocity gradient, respectively. As presented in Figure 4, WSS would be affected considerably under the influence of Emphysema during inhalation. Higher changes for WSS would appear during inhalation time for MV. Hence, Emphysema would cause damage to alveolar walls; observed decreases in WSS values in the balloon part of the alveolar sacs models are rational. As seen in Figure 4, WSS values in the normal alveolar model are larger than the WSS values in the Emphysemic alveolar sac model at the balloon part and the WSS has smaller values at the normal alveolar model's bronchiole part in comparison to the Emphysemic model during inhalation.

\subsection{Pressure and WSS versus Time}

Different planes are defined through models and pressure and WSS curves versus time under mechanical ventilation were plotted and are shown in Figure 5. The human respiratory system is made up of numerous volumes

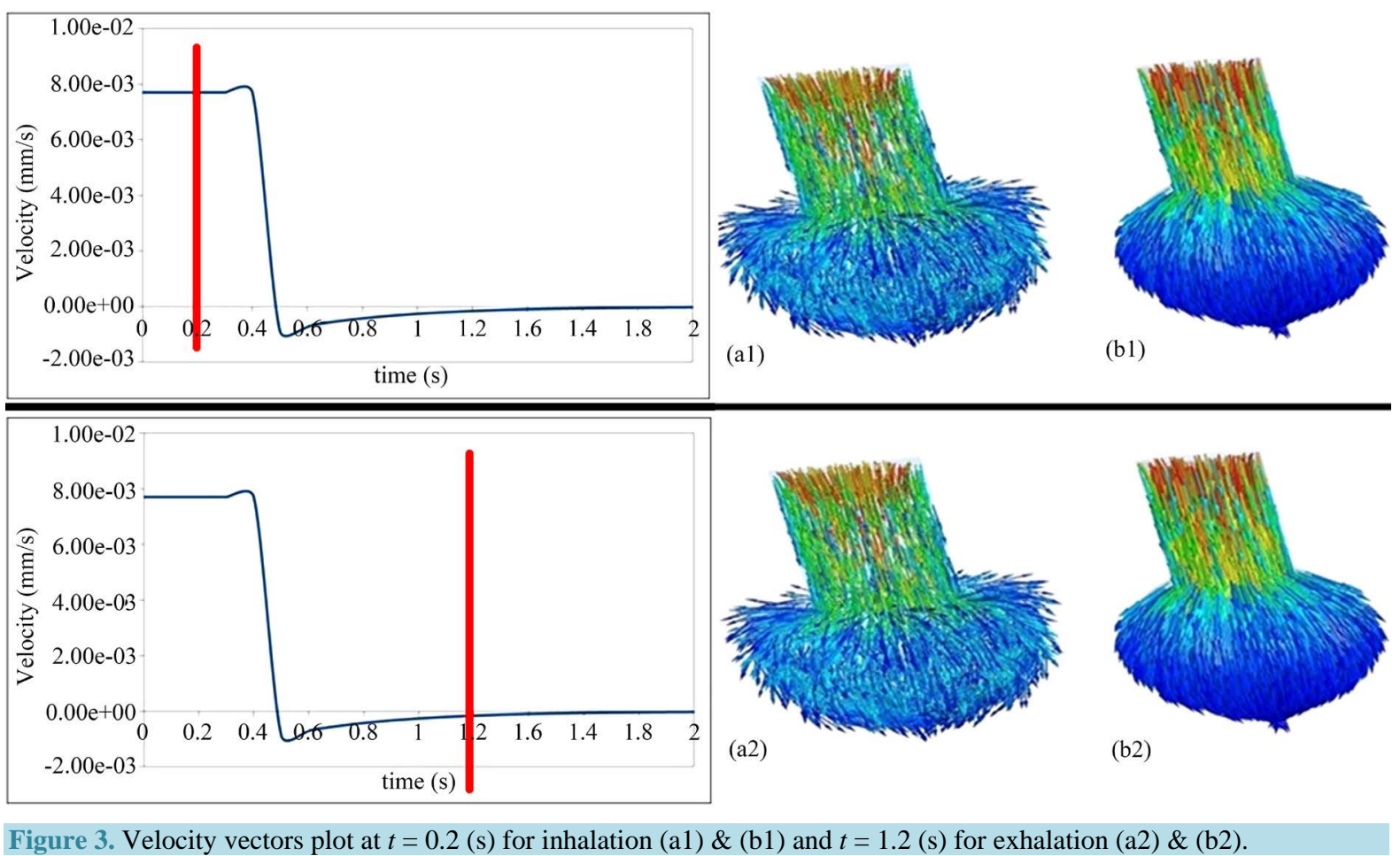



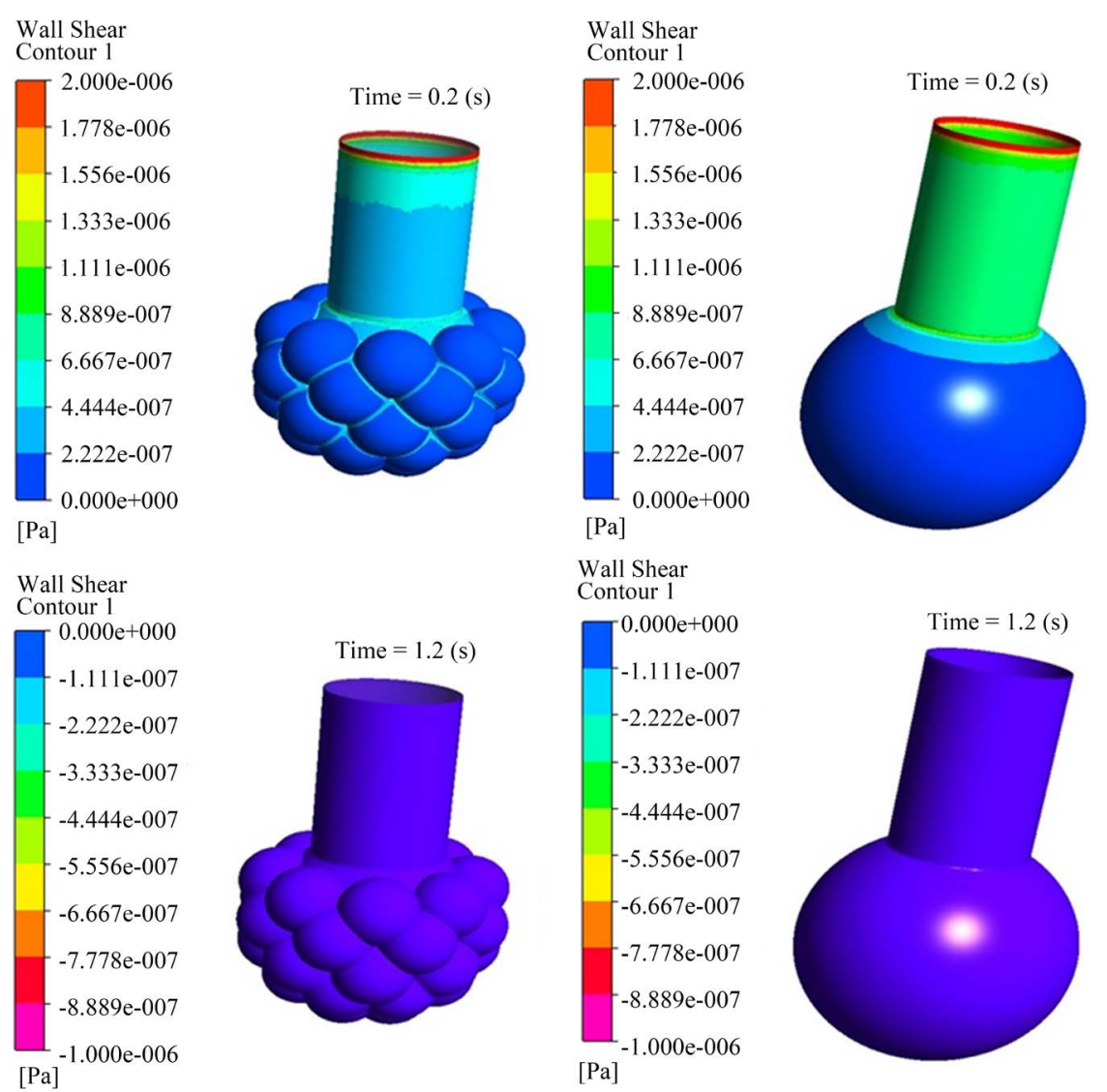

Wall Shear

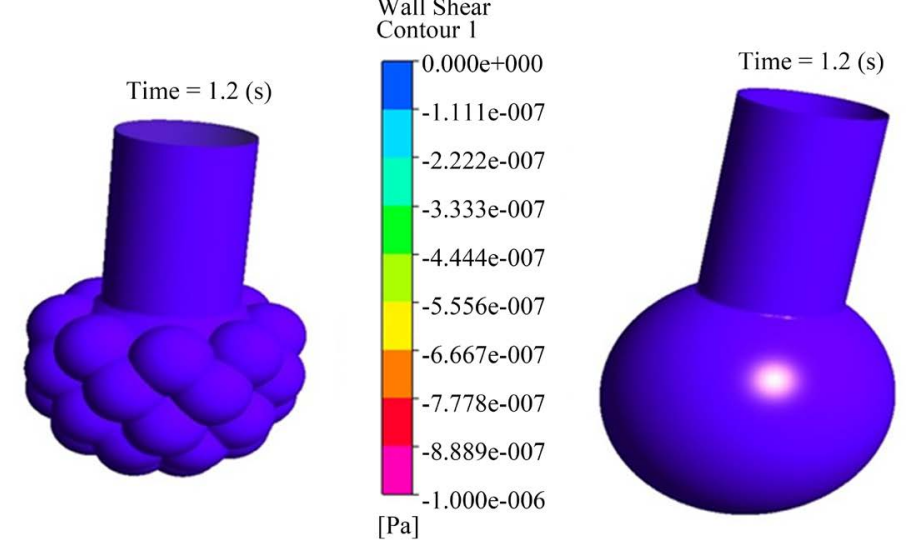

Figure 4. WSS for normal alveolar sac and alveolar sac under influence of Emphysema at $t=0.2$ (s) for inhalation and $t=1.2$ (s) for exhalation.

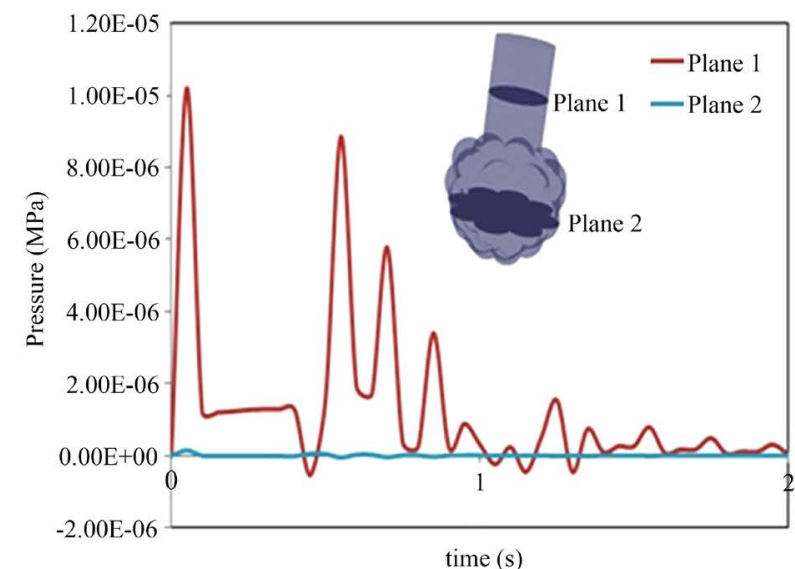

(a)

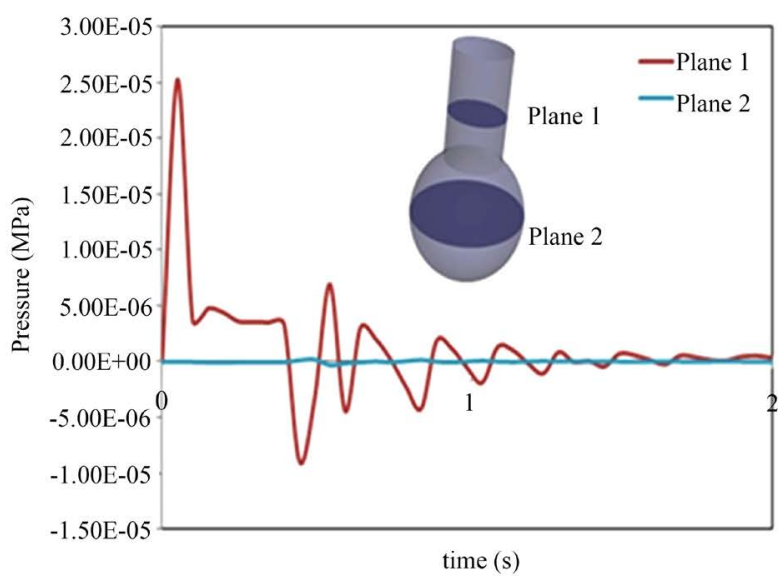

(b)

Figure 5. Pressure-time curves at predefined plane through normal alveolar sac model (a) and model under influence of Emphysema (b).

of air with different pressure values during the respiratory cycle. When the flow is occluded, pressure at different locations is equalized. This equalized pressure multiplied by the total air volume represents total initial pressure-volume. In the case where alveoli have larger volume in comparison to other volumes, the equalized pressure would generally be equal to the existing pressure in the alveoli at the moment of interruption. Pressure equalization during each interruption indicates that the rate of flow suddenly drops or rises to zero, where it re- 
mains during the period of interruption. At the same time, the mouth pressure suddenly goes up and down and oscillates around a slowly rising or falling line [6]. Therefore, the observed pressure changes through the breathing cycle are related to local collapse in the human respiratory system. As discussed earlier, alveolar sac volume would be expected to increase due to the effect of Emphysema. While the alveolar volume increased, it was observed that the average pressure area in the predefined plane was higher under the influence of Emphysema in comparison to the normal alveolar sacs model during mechanical ventilation. There was a significant impact of pressure on inhalation as compared to exhalation.

Moreover, WSS-time curves are presented for both models in Figure 6. As shown in the figure, the Emphysemic model resulted in lower WSS magnitude values during inhalation and higher values during exhalation compared to the normal alveolar sacs model under mechanical ventilation.

\subsection{Collapsed Case Studies}

Different models of collapsed cases were considered to investigate the influence of Influenza viruses on fluid flow. Case (I) considered half of the total volume and Case (II) considered a quarter of the total volume of alveolar sacs balloon geometries to be collapsed at the bottom. Case (III) considered a quarter of the total volume of alveolar sacs balloon geometries to be collapsed at the side. CFD simulations were conducted on all six collapsed models for both normal and Emphysemic alveolar sacs models, and results were post processed. The average pressure area at different locations through the models and WSS through whole model walls were extracted and comparisons were made between area average pressure and WSS results. As illustrated in Figure 7, Figure 8 collapse at half of the volume at the bottom of the alveolar sacs model, caused a decrease in average and maximum pressure values and resulted in higher WSS values for fluid flow during the breathing cycle. Moreover, at a quarter volume collapse at the bottom and side of the model led to higher values for average and maximum pressure and WSS.

\section{Conclusion}

This study investigated the effects of Emphysema and Influenza on alveolar sacs air flow characteristics through a Computational Fluid Dynamics (CFD) simulation. For this purpose, several simulations were conducted on open and collapsed cases for both normal and Emphysemic alveolar sacs models. Results showed Emphysema caused an increase in fluid velocity and applied pressure on alveolar sacs wall and WSS during inhalation. It also led to lower WSS values in alveolar sacs during the exhalation cycle. As hypothesized, alvelolar sac collapse at half of the volume at the bottom of the alveolar sacs models yielded a decrease in average and maximum pressure values and an increase in WSS values for fluid flow during the breathing cycle. Additionally, it was found that a quarter volume collapse led to higher values for average and maximum pressure and WSS values. The influence of Emphysema and influenza virus replication on fluid flow through alveolar sacs models was simultaneously explored and it was shown that these two diseases would generally lead to an increase in fluid pressure. Although alveolar sacs' collapse would cause WSS to rise, a reduction in WSS during inhalation was observed under the influence of Emphysema. The combination of Emphysema and alveolar closure caused a slight increase in average WSS during the breathing cycle with maximum WSS observed during exhalation and

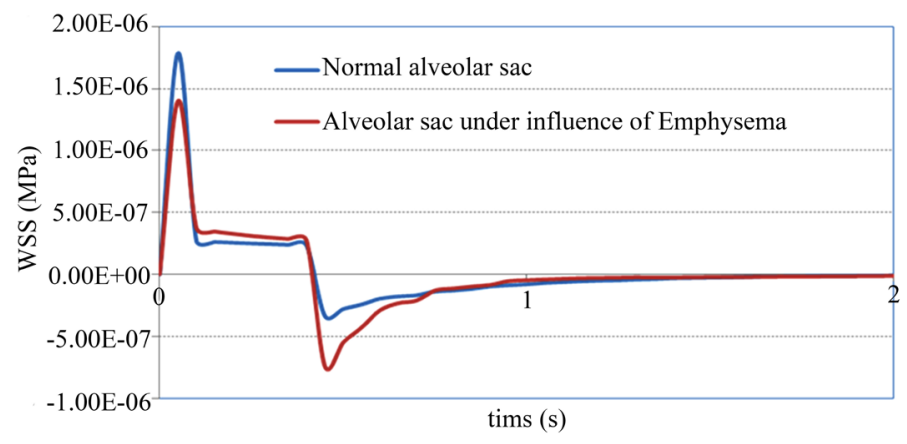

Figure 6. WSS-time curves for normal alveolar sac model and model under influence of Emphysema. 


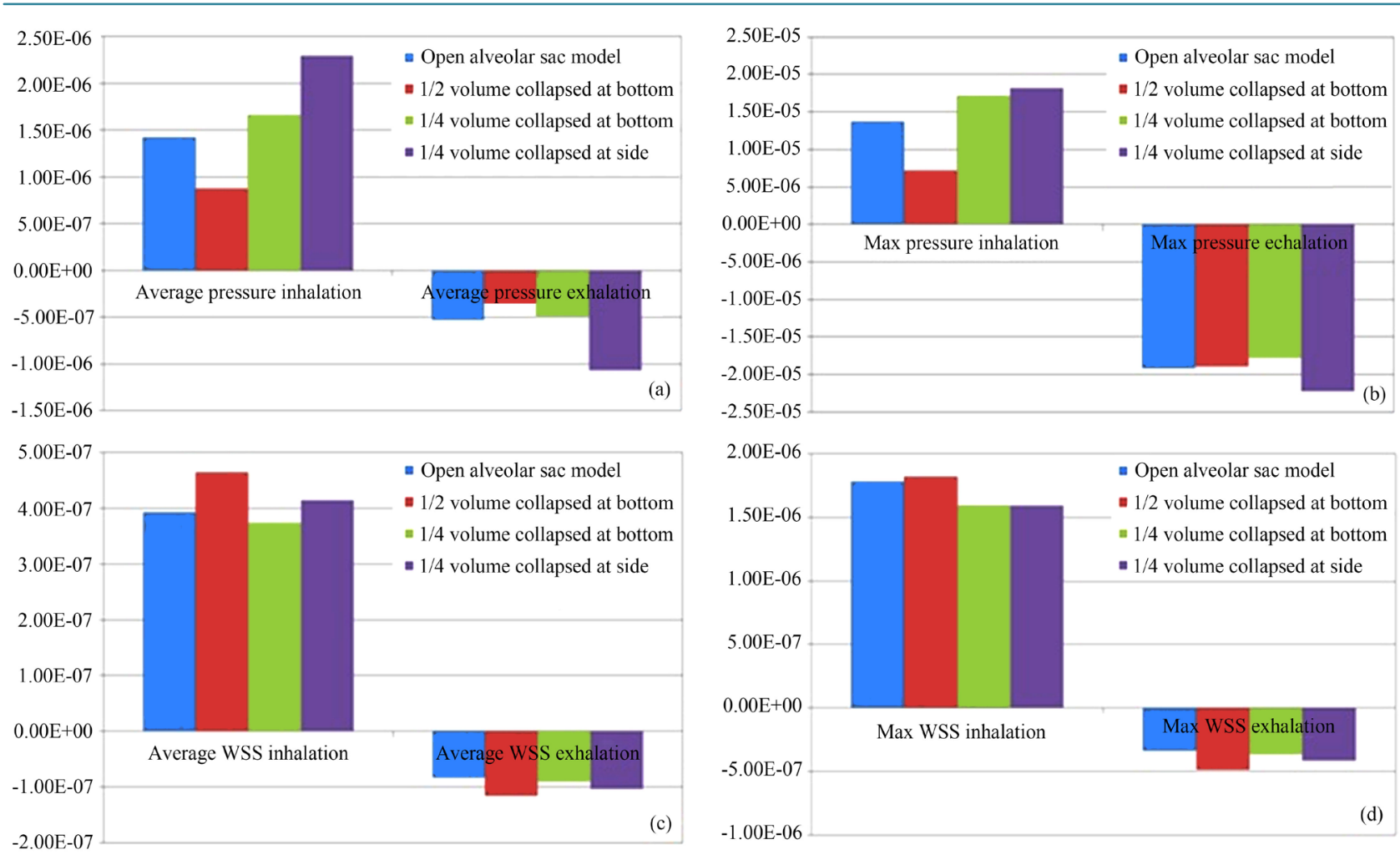

Figure 7. Average and maximum pressure and WSS for breathing cycle for normal alveolar sac model.
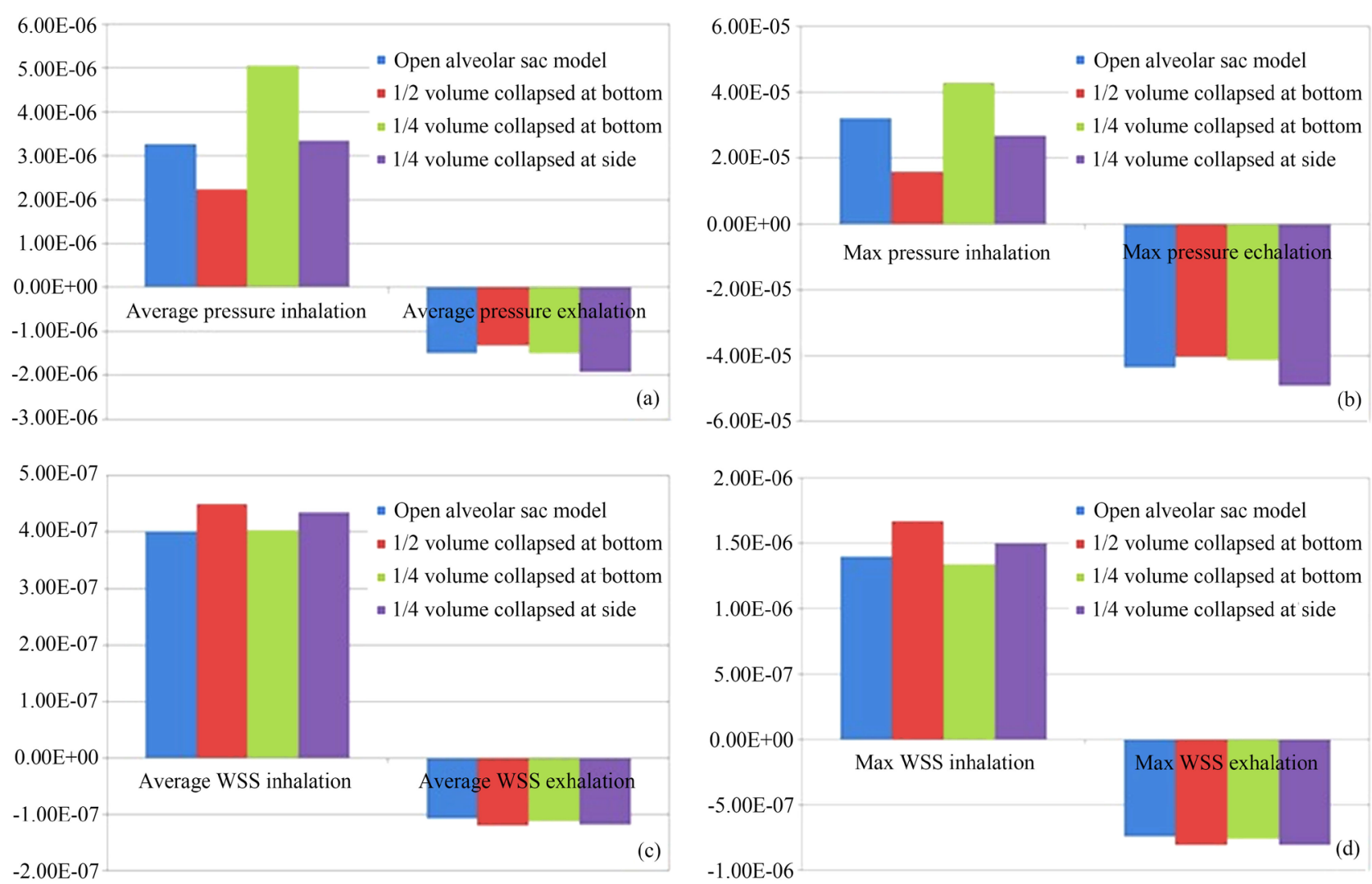

Figure 8. Average and maximum pressure and WSS for breathing cycle for alveolar sac model under influence of Emphysema. 
a decrease in maximum WSS during inhalation. However, more realistic simulations should include the fluidsolid interaction studies. The results obtained provide useful information for respiratory specialists treating patients with diseases such as emphysema and influenza and assist them in developing patient specific guidelines for effective use of mechanical ventilation.

\section{Acknowledgements}

The authors thank the NSF for support this research through a grant CMMI-1430379.

\section{References}

[1] Karl, A., Henry, F.S. and Tsuda, A. (2004) Low Reynolds Number Viscous Flow in an Alveolated Duct. Journal of Biomechanical Engineering, 126, 420-429. http://dx.doi.org/10.1115/1.1784476

[2] Sznitman, J., et al. (2007) Three-Dimensional Convective Alveolar Flow Induced by Rhythmic Breathing Motion of the Pulmonary Acinus. Journal of biomechanical Engineering, 129, 658-665. http://dx.doi.org/10.1115/1.2768109

[3] Henry, F.S., Butler, J.P. and Tsuda, A. (2002) Kinematically Irreversible ACINAR flow: A Departure from Classical Dispersive Aerosol Transport Theories. Journal of Applied Physiology (Bethesda, Md.: 1985), 92, 835-845.

[4] Tsuda, A., Otani, Y. and Butler, J.P. (1999) Acinar Flow Irreversibility Caused by Perturbations in Reversible Alveolar Wall Motion. Journal of Applied Physiology, 86, 977-984.

[5] Darquenne, C., Harrington, L. and Prisk, G.K. (2009) Alveolar Duct Expansion Greatly Enhances Aerosol Deposition: A Three-Dimensional Computational Fluid Dynamics Study. Philosophical Transactions of the Royal Society A: Mathematical, Physical and Engineering Sciences, 367, 2333-2346. http://dx.doi.org/10.1098/rsta.2008.0295

[6] Otis, A.B., Fenn, W.O. and Rahn, H. (1950) Mechanics of Breathing in Man. Journal of Applied Physiology, 2, 592607.

[7] Oakes, J.M., et al. (2010) Flow Field Analysis in Expanding Healthy and Emphysematous Alveolar Models Using Particle Image Velocimetry. Journal of Biomechanical Engineering, 132, 021008. http://dx.doi.org/10.1115/1.4000870

[8] Fitz-Clarke, J.R. (2007) Mechanics of Airway and Alveolar Collapse in Human Breath-Hold Diving. Respiratory Physiology \& Neurobiology, 159, 202-210. http://dx.doi.org/10.1016/j.resp.2007.07.006

[9] Schirrmann, K., et al. (2010) Theoretical Modeling of the Interaction between Alveoli during Inflation and Deflation in Normal and Diseased Lungs. Journal of Biomechanics, 43, 1202-1207. http://dx.doi.org/10.1016/j.jbiomech.2009.11.025

[10] Berg, E.J. and Robinson, R.J. (2011) Stereoscopic Particle Image Velocimetry Analysis of Healthy and Emphysemic Alveolar sac Models. Journal of Biomechanical Engineering, 133, 061004. http://dx.doi.org/10.1115/1.4004251

[11] Hang, D.T.T., et al. (2011) Differential Effect of Prior Influenza Infection on Alveolar Macrophage Phagocytosis of Staphylococcus aureus and Escherichia coli: Involvement of Interferon-Gamma Production. Microbiology and Immunology, 55, 751-759. http://dx.doi.org/10.1111/j.1348-0421.2011.00383.x

[12] Salmon, R., et al. (1981) Human Lung Pressure-Volume Relationships: Alveolar Collapse and Airway Closure. Journal of Applied Physiology, 51, 353-362.

[13] Tsuchida, S., et al. (2006) Atelectasis Causes Alveolar Injury in Nonatelectatic Lung Regions. American Journal of Respiratory and Critical Care Medicine, 174, 279-289. http://dx.doi.org/10.1164/rccm.200506-1006OC

[14] Shields, T.W. (2005) General Thoracic Surgery. Vol. 1. Lippincott Williams \& Wilkins, Philadelphia.

[15] Wall, W.A. and Rabczuk, T. (2008) Fluid-Structure Interaction in Lower Airways of CT-Based Lung Geometries. International Journal for Numerical Methods in Fluids, 57, 653-675. http://dx.doi.org/10.1002/fld.1763

[16] Fung, Y. (1988) A Model of the Lung Structure and Its Validation. Journal of Applied Physiology, 64, 2132-2141.

[17] Kitaoka, H., Nieman, G.F., Fujino, Y., Carney, D., DiRocco, J. and Kawase, I. (2007) A 4-Dimensional Model of the Alveolar Structure. The Journal of Physiological Sciences, 57, 175-185. http://dx.doi.org/10.2170/physiolsci.RP000807

[18] Hansen, J.E., Ampaya, E.P., Bryant, G.H. and Navin, J.J. (1975) Branching Pattern of Airways and Air Spaces of a Single Human Terminal Bronchiole. Journal of Applied Physiology, 38, 983-989.

[19] Kumar, H., Tawhai, M.H., Hoffman, E.A. and Lin, C.-L. (2009) The Effects of Geometry on Airflow in the Acinar Region of the Human Lung. Journal of Biomechanics, 42, 1635-1642. http://dx.doi.org/10.1016/j.jbiomech.2009.04.046

[20] Gehr, P., Bachofen, M. and Weibel, E.R. (1978) The Normal Human Lung: Ultrastructure and Morphometric Estimation of Diffusion Capacity. Respiration Physiology, 32, 121-140. http://dx.doi.org/10.1016/0034-5687(78)90104-4 
[21] Berg, E.J., Weisman, J.L., Oldham, M.J. and Robinson, R.J. (2010) Flow Field Analysis in a Compliant Acinus Replica Model Using Particle Image Velocimetry (PIV). Journal of Biomechanics, 43, 1039-1047. http://dx.doi.org/10.1016/j.jbiomech.2009.12.019

[22] Tsuda, A., Rogers, R.A., Hydon, P.E. and Butler, J.P. (2002) Chaotic Mixing Deep in the Lung. Proceedings of the National Academy of Sciences of the United States of America, 99, 10173-10178. http://dx.doi.org/10.1073/pnas.102318299

[23] Anjilvel, S. and Asgharian, B. (1995) A Multiple-Path Model of Particle Deposition in the Rat Lung. Fundamental and Applied Toxicology, 28, 41-50. http://dx.doi.org/10.1006/faat.1995.1144

[24] Barth, T.J. and Jespersen, D.C. (1989) The Design and Application of Upwind Schemes on Unstructured Meshes. 27th Aerospace Sciences Meeting, Reno, 9-12 January 1989, 13 p.

[25] Butler, J.P. and Tsuda, A. (2005) Logistic Trajectory Maps and Aerosol Mixing Due to Asynchronous Flow at Airway Bifurcations. Respiratory Physiology \& Neurobiology, 148, 195-206. http://dx.doi.org/10.1016/j.resp.2005.06.001

[26] Aris, R. (1956) On the Dispersion of a Solute in a Fluid Flowing through a Tube. Proceedings of the Royal Society A: Mathematical, Physical and Engineering Sciences, 235, 67-77. http://dx.doi.org/10.1098/rspa.1956.0065

[27] Sturm, R. and Hofmann, W. (2004) Stochastic Simulation of Alveolar Particle Deposition in Lungs Affected by Different Types of Emphysema. Journal of Aerosol Medicine, 17, 357-372. http://dx.doi.org/10.1089/jam.2004.17.357

[28] Sznitman, J., Heimsch, T., Wildhaber, J.H., Tsuda, A. and Rösgen, T. (2009) Respiratory Flow Phenomena and Gravitational Deposition in a Three-Dimensional Space-Filling Model of the Pulmonary Acinar Tree. Journal of Biomechanical Engineering, 131, Article ID: 031010. http://dx.doi.org/10.1115/1.3049481

[29] Weibel, E. (1964) Morphometrics of the Lung, in Handbook of Physiology Respiration. American Physiological Society, Washington DC, 285-307.

[30] Klingele, T.G. and Staub, N.C. (1970) Alveolar Shape Changes with Volume in Isolated, Air-Filled Lobes of Cat Lung. Journal of Applied Physiology, 28, 411-414.

[31] Issa, R.I. (1986) Solution of the Implicitly Discretised Fluid Flow Equations by Operator-Splitting. Journal of Computational Physics, 62, 40-65. http://dx.doi.org/10.1016/0021-9991(86)90099-9

[32] Liu, Y., So, R.M.C. and Zhang, C.H. (2002) Modeling the Bifurcating Flow in a Human Lung Airway. Journal of Biomechanics, 35, 465-473. http://dx.doi.org/10.1016/S0021-9290(01)00225-1

[33] Fluent, A. (2011) 14.0 Tutorial Guide. ANSYS Inc., Canonsburg.

[34] Detmer, S.E. (2015) Pathogenesis of Influenza a Virus Infection in Swine. https://www.pig333.com/swine-influenza/pathogenesis-of-influenza-a-virus-infection-in-swine_9635/ 